\title{
Changes of lymphatic flow caused by core needle biopsy of axillary sentinel lymph node in a rabbit model
}

\author{
Yiqin Xia ${ }^{1 \#}$, Mingjie Zheng ${ }^{1 \#}$, Lie Chen ${ }^{1}$, Yangyang Cui ${ }^{1}$, Huaxing Huang ${ }^{2}$, Peng Kong ${ }^{1}$, Wenbin Zhou ${ }^{1}$, \\ Hui Xie ${ }^{1}$, Shui Wang ${ }^{1}$ \\ ${ }^{1}$ Department of Breast Surgery, the First Affiliated Hospital, Nanjing Medical University, Nanjing, China; ${ }^{2}$ The First Clinical Medical College, \\ Nanjing Medical University, Nanjing, China \\ Contributions: (I) Conception and design: Y Xia, M Zheng, W Zhou, H Xie; (II) Administrative support: W Zhou; (III) Provision of study materials \\ or patients: Y Xia, M Zheng, L Chen, Y Cui, H Huang, P Kong, W Zhou, H Xie, S Wang; (IV) Collection and assembly of data: Y Xia, M Zheng, \\ L Chen, Y Cui, H Huang, P Kong, W Zhou, H Xie, S Wang; (V) Data analysis and interpretation: Y Xia, M Zheng; (VI) Manuscript writing: All \\ authors; (VII) Final approval of manuscript: All authors. \\ \#These authors contributed equally to this work. \\ Correspondence to: Wenbin Zhou, Hui Xie. Department of Breast Surgery, The First Affiliated Hospital, Nanjing Medical University, Nanjing 210029 , \\ China. Email: zhouwenbin@njmu.edu.cn; Hxie@njmu.edu.cn.
}

\begin{abstract}
Background: Core needle biopsy (CNB) plays an important role in the preoperative axillary lymph node (ALN) assessment in breast cancer (BC) patients with the development of treatment, but little is known about the axillary lymph flow after CNB of ALNs. This study aimed to investigate the changes of lymphatic flow after CNB of sentinel lymph node (SLN) in a rabbit model.

Methods: The axillary SLN was biopsied in a rabbit model, and the changes of sentinel lymph flow were observed by methylthioninium chloride imaging at 1 and 12 days after the biopsy. Furthermore, the afferent lymphatic vessel was ligated and imaged once every 3 days to assess the changes of lymphatic flow.

Results: The SLN biopsied was characterized by disorganized medullary sinus containing erythrocytes, whereas clean medullary sinus containing a normal population of circulating lymphoid cells was observed in the contralateral normal SLN. At 1 day after biopsy, the sentinel lymphatic drainage was blocked. At 12 days after biopsy, the sentinel lymphatic flow was reconstructed or repaired. Ligation of afferent lymphatic vessel further confirmed the reconstruction of lymphatic flow.
\end{abstract}

Conclusions: The sentinel lymphatic flow changes after CNB in a rabbit model, but it can be reconstructed or repaired.

Keywords! Breast cancer (BC); sentinel lymph node (SLN); core needle biopsy (CNB); lymphatic flow

Submitted Apr 02, 2020. Accepted for publication Sep 10, 2020.

doi: $10.21037 /$ apm-20-882

View this article at: http://dx.doi.org/10.21037/apm-20-882

\section{Introduction}

With the development of sentinel lymph node biopsy (SLNB), the use of axillary surgery has been progressively reduced in patients with early-stage breast cancer (BC), which results in fewer patients undergoing axillary lymph node dissection (ALND). Currently, SLNB has been widely accepted as a standard of care, with lower surgical morbidity, including lymphedema, shoulder stiffness, pain, and paraesthesia as compared to ALND. Long-term followup shows that the incidence of lymphedema is as high as $40 \%$ after BC treatment (1). However, lymphedema still occurs in $7 \%$ of patients undergoing SLNB (2). Lymphedema severely affects the quality of life of BC patients due to the lifestyle and occupational alternations, change in the functional status, as well as changes in the psychosocial and economic aspects (3). The clinical signs vary with the duration and severity of lymphedema, from vague 
sensations of limb heaviness to structural distortion (1). In the past 5-10 years, more attention has been paid to minimizing the morbidity after surgical axillary treatment.

Under the guidance of contrast-enhanced ultrasound (CEUS), the ALNs can be identified and biopsied preoperatively (4). A preoperative biopsy proved axillary metastasis may serve as an indication to neoadjuvant chemotherapy or ALND (5). SLNB is still needed for patients with negative biopsy results to rule out falsenegative results, although the false-negative may occur because the low-volume disease within the sentinel lymph nodes (SLN) (5). Preoperative biopsy may spare some patients from directly SLNB.

Preoperative lymph node biopsy can be done with either core needle biopsy $(\mathrm{CNB})$ or fine needle aspiration biopsy (FNAB). The image-guided CNB has been a diagnostic procedure for breast lesions, and has replaced FNAB due to its higher sensitivity (6). It therefore is hypothesized that $\mathrm{CNB}$ is superior to FNAB for the detection of axillary metastasis. A prospective analysis comparing these two techniques shows that $\mathrm{CNB}$ is more sensitive when accurate preoperative axillary staging is needed (7). Moreover, immunohistochemical technique may be added to CNB to get further information on the disease.

However, little is known about the axillary lymph flow after CNB of ALNs. In a mouse model, results showed the lymph flow in the collecting lymphatic vessels significantly changed after lymph node dissection, and long term interruption of lymph flow might cause lymphatic dysfunction, contributing to lymphedema (8). It is assumed that CNB may cause similar damage to the axillary lymphatic vessels. Thus, this study was undertaken to investigate the changes of lymphatic flow after CNB of axillary SLN in a rabbit model. We present the following article in accordance with the ARRIVE reporting checklist (available at http://dx.doi.org/10.21037/apm-20-882).

\section{Methods}

\section{Study design}

Twenty-two rabbits were divided into three groups. There were 2 rabbits in the Group 1, 14 in the Group 2A, 3 in the Group 2B and 3 in the Group 3. The hair on the chest were removed before methylthioninium chloride imaging. Then, $0.1 \mathrm{~mL}$ of methylthioninium chloride (Jichuan, Inc.) was injected intracutaneously around the left areola with a 26-gauge needle, and the sentinel lymphatic flow was imaged. In the Group 1 and Group 2A, core biopsy of the left axillary SLN with a 14-gauge biopsy gun (MG12-22, Bard, Inc.) was performed immediately after the injection. In the Group 1, the left axillary SLN was immediately harvested after the biopsy, and the contralateral SLN of 2 rabbits was harvested without biopsy using the same imaging method.

In the Group 2A, 6-0 Proline (Johnson\&Johnson, Inc.) was used to mark the biopsied SLN in 14 rabbits. At 1 day after and 12 days after the biopsy, methylthioninium chloride imaging was performed to observe the lymphatic flow.

Group 2B served as a sham-operation group of Group 2A. All the experimental procedures were consistent with Group 2A except that the SLN was not biopsied.

In the Group 3, the afferent lymphatic vessel was ligated with 6-0 suture immediately after the injection to artificially block the drainage of methylthioninium chloride. The rabbits were imaged once every 3 days after the ligation, and the lymphatic flow was observed.

\section{Animal model}

This study was approved by the Institutional Animal Care and Use Committee of Nanjing Medical University (No.: 1601179), in compliance with the Animal Ethical and Welfare Committee (AEWC) of China. Female New Zealand white rabbits (age: 12-14 weeks) weighing $2.0-2.5 \mathrm{~kg}$ were used in this study. All animal studies were performed by trained investigators with experience in CNB. For all experiments and procedures, anesthesia was induced by intravenous injection of chloral hydrate $(3 \mathrm{~mL} / \mathrm{kg})$. Animals that refused solid and fluid intake for more than 4 days with concomitant apathy and weight loss of more than $20 \%$ were sacrificed and not included in the final analysis.

\section{Core biopsy}

The core biopsy was performed by directly visualizing the SLN and corresponding afferent lymphatic vessel. The biopsy gun was held perpendicular to the afferent lymphatic vessel and parallel to the chest wall of the rabbit.

\section{Pathology}

All specimens were fixed in $10 \%$ paraformaldehyde and embedded in paraffin. 5 - $\mu \mathrm{m}$ sections were obtained and stained with haematoxylin and eosin (H\&E). All histologic examinations were performed by one pathologist. 

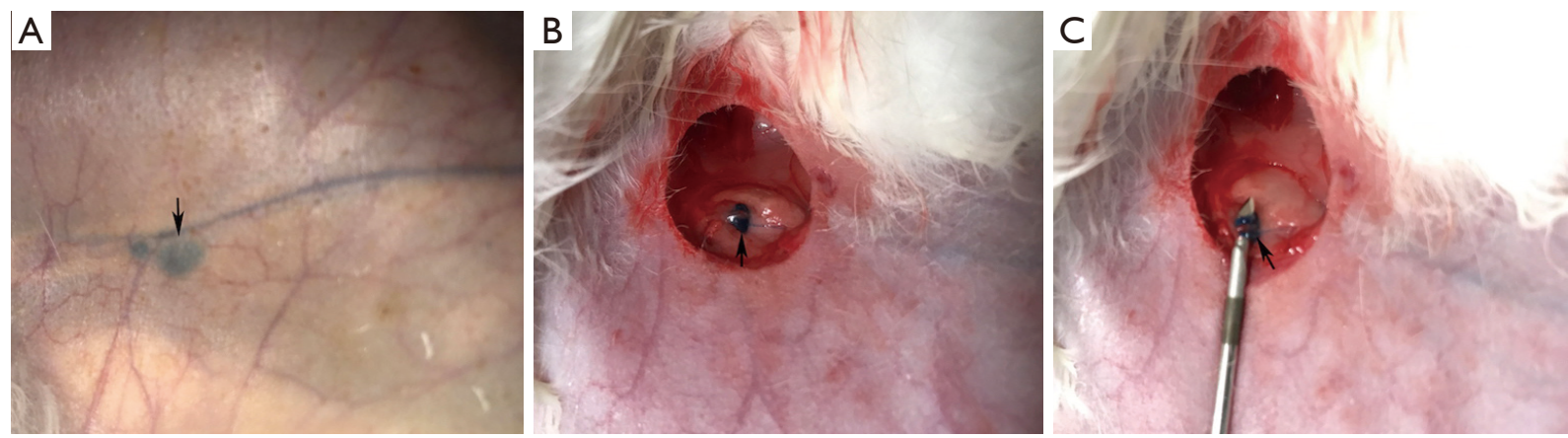

Figure 1 Imaging after areola intracutaneous methylene blue injection. Stained SLN (A), SLN (arrow) after skin incision (B) and direction of the core needle (C). SLN, sentinel lymph node.

\section{Results}

\section{Sentinel lymphatic flow changed after CNB of SLN}

Methylthioninium chloride was injected intracutaneously around the areola and the SLN was detected in the axilla (Figure 1A). Subsequently, a small incision overlying the SLN was made to expose the SLN and corresponding afferent lymphatic vessel (Figure $1 B$ ). The SLN was biopsied using a core needle perpendicular to the afferent lymphatic vessel and parallel to the chest wall of the rabbit (Figure 1C). After the biopsy, in the Group 1, the SLN was harvested and compared with the normal contralateral SLN without biopsy. The SLN biopsied was characterized by a disorganized medullary sinus containing erythrocytes (Figure $2 A$ ), whereas clean medullary sinus containing a normal population of circulating lymphoid cells was observed in the contralateral normal SLN (Figure 2B). Moreover, the afferent lymphatic vessel in the SLN biopsied seemed to be more distended than in the contralateral normal SLN (Figure 2C,D).

In the Group 2A, of the 14 axillaries, sentinel lymphatic flow was well defined in 12 . Of 12 axillaries, 2 SLNs were found in 2 axillaries; only one SLN was found in the remaining 10 axillaries. In all, biopsy was performed in 14 lymph nodes. At 1 and 12 days after biopsy, methylthioninium chloride imaging was performed. The lymphatic flow was defined to be unblocked when the SLN was totally or partially stained (Figure $3 A, B$ ) but blocked when the SLN was not stained or only the afferent lymphatic vessel was stained (Figure 3C,D). The lymphatic flow after CNB is shown in Table 1. At one day after biopsy, 8 of 14 SLN was blocked on the methylthioninium chloride imaging, of which neither SLN nor the afferent lymphatic vessel was stained in 6 animals, while only afferent lymphatic vessel was stained in another 2 animals. There were 4 animals with similar staining as before biopsy. Interestingly, at 12 days after CNB, the drainage of 3 SLN was blocked on the methylthioninium chloride imaging. At the same time, the lymphatic drainage was not blocked in 6 animals. In the sham-operation group, sentinel lymphatic flow was all well defined in 3 ALNs, and the lymphatic drainage remained unchanged at 1 and 12 days after shamoperation (Figure 3E,F).

\section{Sentinel lymphatic flow was reconstructed or repaired after SLN biopsy}

The lymphatic flow was imaged in 7 animals at 1 and 12 days after biopsy (Table 1). The lymphatic flow in 5 animals was blocked at 1 day after biopsy, in which lymphatic flow was still blocked in 1 animal at 12 days after biopsy. The lymphatic flow in 4 animals was blocked at 1 day after CNB, but unblocked at 12 days, and drainage reconstruction was observed in 3 animals of them. At 12 days after CNB, the former biopsied SLN together with the corresponding afferent lymphatic vessel failed to be stained. Instead, another lymphatic vessel connecting the injection site to another lymph node was seen (Figure $4 A, B, C$ ). In an animal with lymphatic flow repair, the biopsied SLN was not stained at 1 day after CNB but the same SLN with corresponding afferent lymphatic vessel was found to be filled with methylene blue at 12 days after CNB (Figure 4D,E,F).

In another 2 animals, the lymphatic flow was unblocked at 1 day after $\mathrm{CNB}$, of which 1 was still unblocked at 12 days after CNB. However, the lymphatic flow in a remaining animal was blocked at 12 days after $\mathrm{CNB}$, which might be ascribed to the edema of surrounding tissues caused by biopsy. 

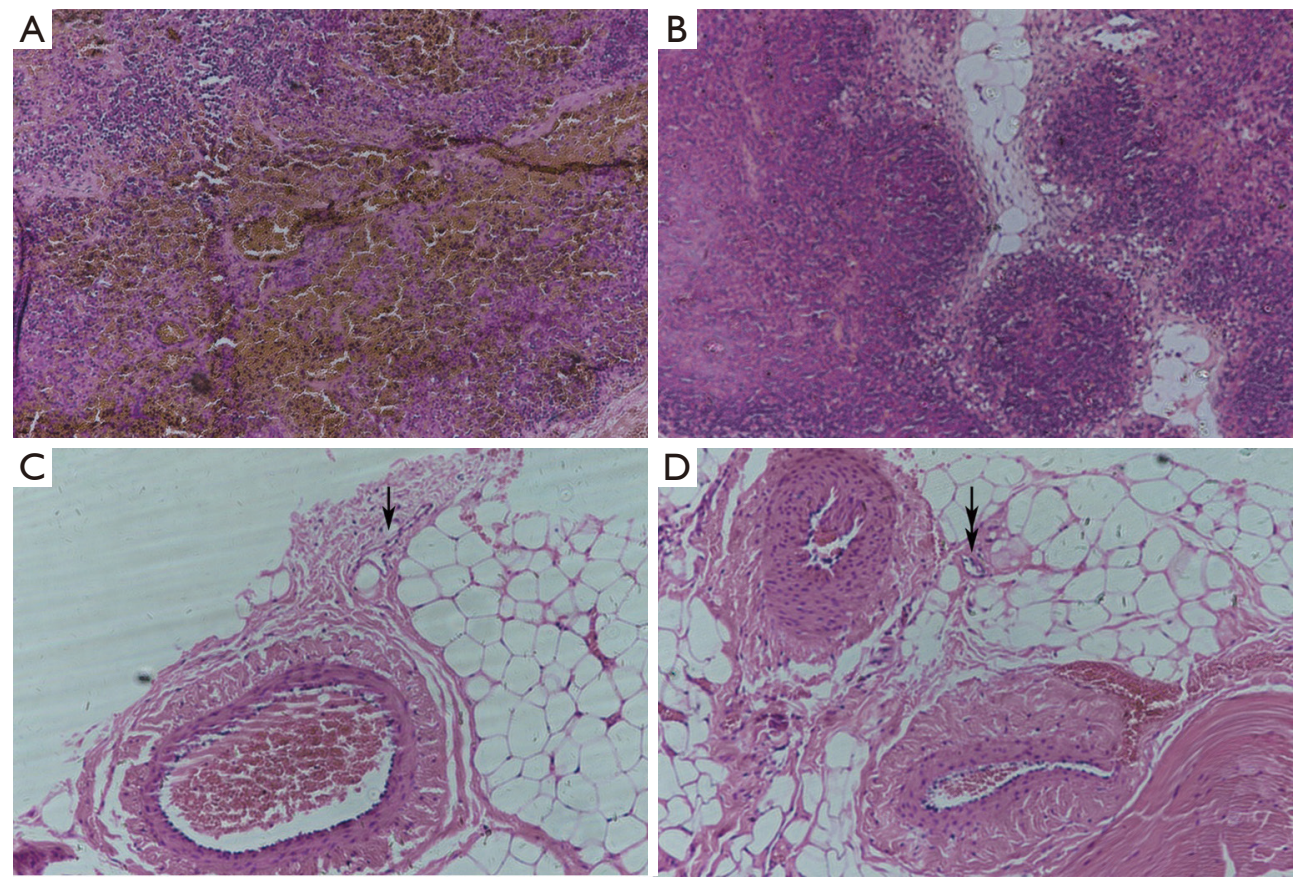

Figure 2 Disorganized medullary sinus containing erythrocytes was found in the biopsied SLN (A, $\times 100)$, whereas clean medullary sinus containing a normal population of circulating lymphoid cells was observed in the contralateral normal SLN (B, $\times 100)$. Afferent lymphatic vessel of the biopsied SLN seemed to be more distended than that at the contralateral side (C,D, $\times 200)$. Arrow: afferent lymphatic vessel of the biopsied SLN. Double arrow: afferent lymphatic vessel of the normal SLN. SLN, sentinel lymph node.
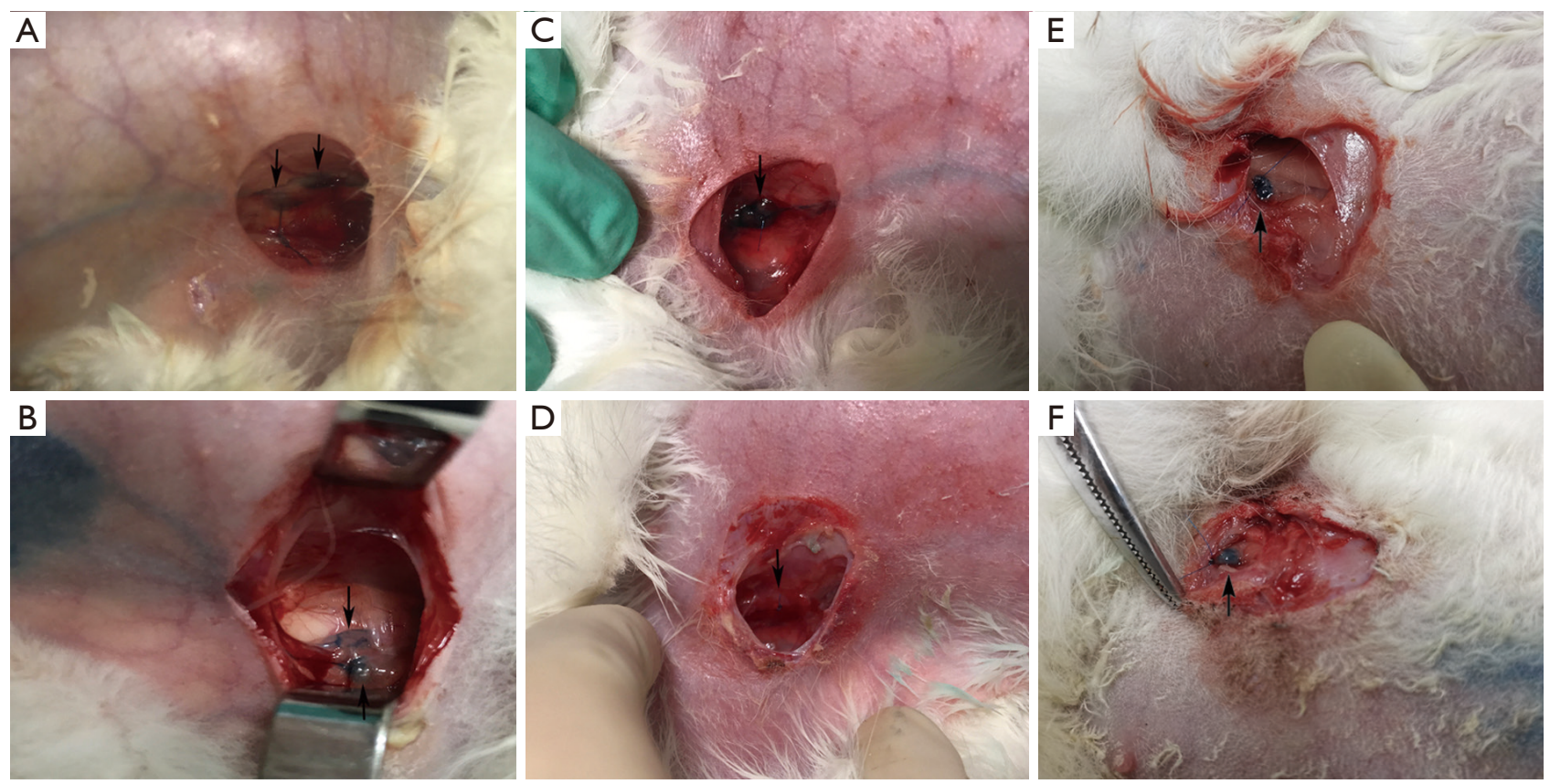

Figure 3 The lymphatic flow was defined to be unblocked when the SLN was totally or partially stained (A,B) but blocked when the SLN was not stained or only the afferent lymphatic vessel was stained (C,D). In the sham-operation group, the lymphatic drainage remained unchanged at 1 and 12 days after sham-operation (E,F). SLN, sentinel lymph node. 
Table 1 The changes in the lymphatic flow after CNB of the SLN at 1 and 12 days post biopsy

\begin{tabular}{lcc}
\hline No. & 1-day post biopsy & 12-day post biopsy \\
\hline 1 & Unblocked & NA \\
2 & Unblocked & Blocked \\
3 & Blocked & NA \\
4 & Blocked & Unblocked \\
5 & Blocked & NA \\
6 & Blocked & Unblocked \\
7 & Blocked & Unblocked \\
8 & Unblocked & NA \\
9 & Unblocked & Unblocked \\
10 & Blocked & NA \\
11 & Blocked & Unblocked \\
12 & Blocked & Blocked \\
13 & NA & Unblocked \\
14 & NA & Blocked \\
\hline
\end{tabular}

CNB, core needle biopsy; SLN, sentinel lymph node.

\section{Validation of lymphatic flow reconstruction after ligation of afferent lymphatic vessel}

In the Group 3, the afferent lymphatic vessels of the SLN were artificially blocked, aiming to confirm the reconstruction of lymphatic flow after biopsy. A torturous, dilated methylthioninium chloride-filled lymphatic vessel was observed at the upstream of the ligated area immediately after ligation. At 1 day after ligation, the ligated lymphatic vessel and the draining SLN were no longer stained by methylthioninium chloride. However, in 3 animals, at as early as 3 days after CNB, another lymphatic vessel connecting the injection site to a new SLN was stained and observed (Figure 5).

\section{Discussion}

To the best of our knowledge, the effect of CNB of the SLN on the sentinel lymphatic flow has not been reported in available studies. Our results showed that core biopsy of the rabbit axillary SLN could dramatically change the initial lymph drainage. Two patterns resulted from the biopsy: reconstruction or repair. Reconstruction means the rerouting of the sentinel lymphatic flow, while repair means the restoration of the same SLN. Hall et al. made a flank incision on the left side of mice to interrupt normal lymphatic flow. They found that newly built lymphatic vessels were detectable from 7-8 days up to 2-3 weeks after injury (9). Kown et al. dissected a single ALN in a mouse model and the functional change in the inguinal-to-axillary lymph drainage was investigated (8). At 2 days after the surgery, no lymph flow was observed, while at 6 days after the surgery, altered lymphatic flow pattern was identified. Eventually, at 9 days after the surgery, there was an ordered lymphatic flow pattern. The phenomenon identified in their study was consistent with the "reconstruction" defined in our study. Sweet et al. demonstrated that lymph flow acted on the valve formation and collecting lymphatic vessel maturation and development (10). Mebius et al. found that lymphatic flow could affect the functional integrity of lymph nodes (11). We speculate that when the biopsy causes damage to the SLN, the slow filling of lymph promotes the restoration of the biopsied SLN, and eventually the "reparation" is present.

In clinical practice, if a benign pathological diagnosis is obtained from the preoperative axillary biopsy, it will be confirmed by the intraoperative SLNB. Thus, CNB of ALNs has an effect on the false-negative rate of SLNB. Studies have found several factors contributing to the falsenegative rate of SLNB, which is about $10 \%$ (12). Great tumor burden in the SLN is one of the factors. Proulx et al. initially found the rerouting of lymphatic flow caused by lymphatic dysfunction, which was induced by the tumor metastatic to the SLN (13). Suga et al. then observed the rerouting in $\mathrm{BC}$ patients by computed tomographic lymphography (14). In a clinical trial, Zhou et al. raised a hypothesis: BC cells migrate to the SLN and the growth of cancer cells might induce lymphatic dysfunction (15). At this time, rerouting of lymph flow occurs. They also found that there were several lymphatic vessels connecting the injected site and different lymph nodes. The afferent lymphatic vessel of the SLN is dominant. Once it is blocked, another lymphatic vessel may act as the dominant drainage path. The phenomenon observed in the present study was consistent with Zhou's hypothesis.

A number of clinical trials have compared $\mathrm{CNB}$ with FNAB, but the results were controversial. Rautiainen $e t$ al. found that the sensitivities of FNAB and CNB were $72.5 \%$ and $88.2 \%$, respectively $(\mathrm{P}=0.008)$ and they speculated that $\mathrm{CNB}$ was significantly more sensitive than FNAB (7). However, a meta-analysis indicated no difference between 

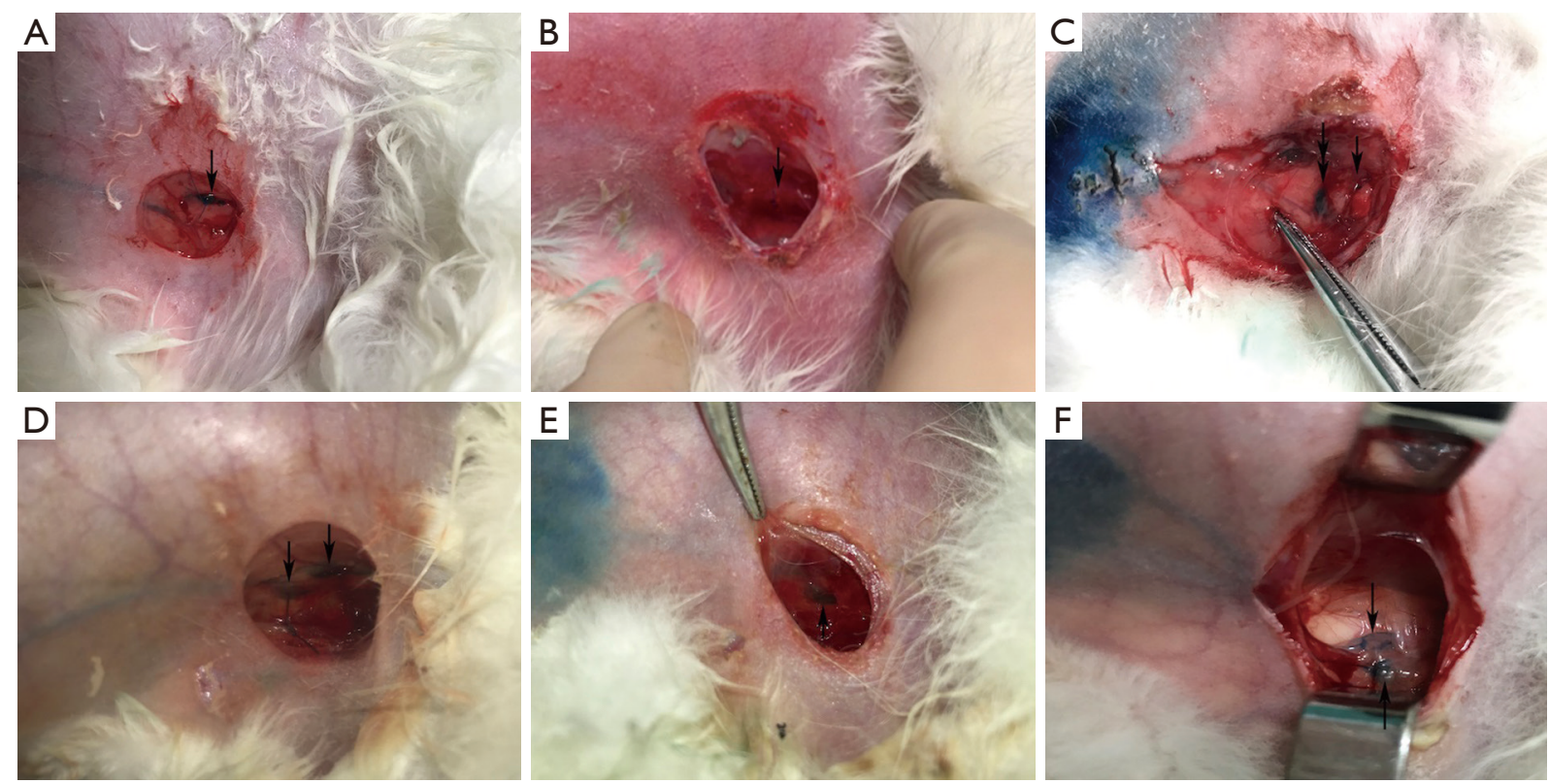

Figure 4 Reconstructed (A,B,C) and repaired (D,E,F) sentinel lymphatic drainage after CNB of the SLN. Arrow: SLN. Double arrow: reconstructed SLN. CNB, core needle biopsy; SLN, sentinel lymph node.
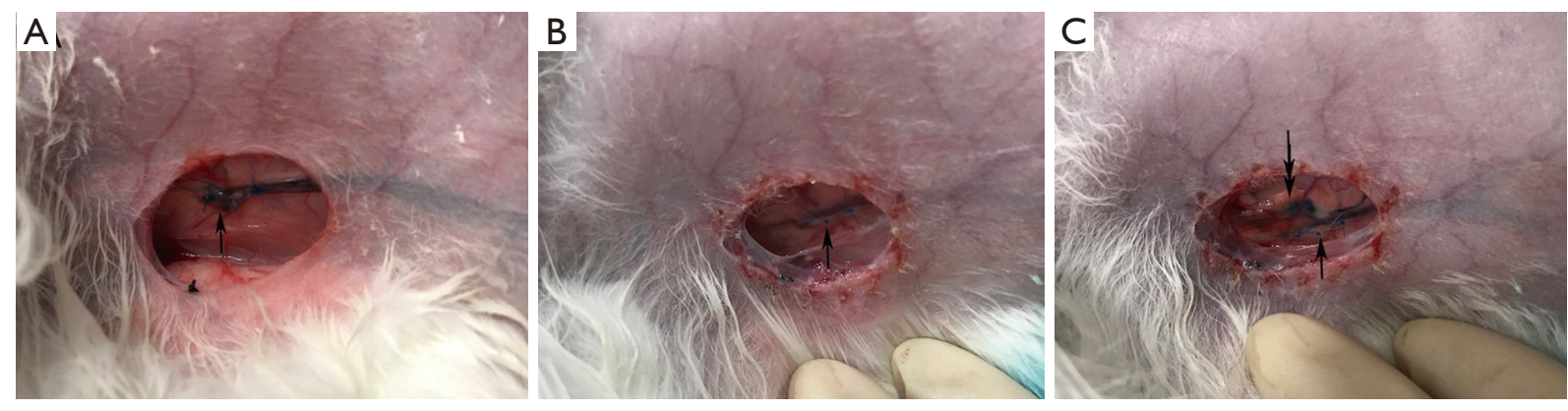

Figure 5 The afferent lymphatic vessel of the SLN (arrow) was ligated (A,B). A reconstructed SLN (double arrow) was observed at 3 days after ligation (C). SLN, sentinel lymph node.

$\mathrm{CNB}$ and FNAB. CNB has some advantages over FNAB (16). Our results showed that CNB of the SLN had its disadvantages. The damage to the lymphatic drainage could not be neglected. Especially, when the SLN has a small size, CNB may not be a tool of choice. In this aspect, FNAB has its advantages. Besides its low cost and convenience, it is less likely to damage the lymphatic flow for its smaller diameter. The controversy mainly focuses on the relatively higher false-negative rate, which may be ascribed to the presence of inadequate samples, misidentification of SLN and low tumor burden. Onsite cytological support helps reduce the incidence of inadequate samples (17). New imaging techniques, including sonoelastography, contrast-enhanced ultrasonography, multispectral optoacoustic tomography (MSOT) and near infrared light fluorescence imaging, make the identification of SLN easier (18). For clinical T1-2N0 BC patients who received breast-conserving surgery, further ALND has no survival benefit for those with 1-2 positive SLNs (19). Liang et al. found that BC patients in whom ultrasonography showed a suspicious lymph node but FNAB showed negative had a lower tumor burden and could be treated as node-negative disease (20).

Our study provides direct evidence that $\mathrm{CNB}$ of the SLN can dramatically change the sentinel lymphatic flow pattern in a rabbit model. We speculate that the physical 
trauma caused by preoperative CNB of the SLN can similarly lead to the dysfunction of the regional lymphatic drainage in clinical practice. Thus, the false-negativerate of intraoperative SLNB may be increased. In this circumstance, CNB is not a best option. Instead, FNAB might be better.

There were still limitations in the present study. First, the reconstruction or repair of the sentinel lymphatic flow was observed. However, the specific mechanism of lymphangiogenesis was not further investigated. Second, the rabbit ALNs without tumor burden were biopsied. Future studies are needed to confirm our findings in animal tumor models. Third, the size of lymph nodes was different between rabbits and humans, especially those with tumor burden. Our ongoing study will confirm these findings.

In conclusion, the sentinel lymphatic flow pattern may be significantly altered by CNB of the SLN. The blocked sentinel lymphatic flow will be restored by reconstruction or repair. More clinical studies are needed to confirm our findings.

\section{Acknowledgments}

Funding: This study was supported by the National Natural Science Foundation of China (No. 81672612 and 81572607), the Project of Philosophy and Social Science Research in Colleges and Universities in Jiangsu Province (No. 2018SJA0276) and Talent Introduction Fund of Nanjing Medical University (No. 2017RC08).

\section{Footnote}

Reporting Checklist: The authors have completed the ARRIVE reporting checklist. Available at http://dx.doi. org/10.21037/apm-20-882

Data Sharing Statement: Available at http://dx.doi. org/10.21037/apm-20-882

Conflicts of Interest: All authors have completed the ICMJE uniform disclosure form (available at http://dx.doi. org/10.21037/apm-20-882). The authors have no conflicts of interest to declare.

Ethical Statement: The authors are accountable for all aspects of the work in ensuring that questions related to the accuracy or integrity of any part of the work are appropriately investigated and resolved. The experiments were approved by the Institutional Animal Care and Use Committee of Nanjing Medical University (No.: 1601179), in compliance with the Animal Ethical and Welfare Committee (AEWC) of China.

Open Access Statement: This is an Open Access article distributed in accordance with the Creative Commons Attribution-NonCommercial-NoDerivs 4.0 International License (CC BY-NC-ND 4.0), which permits the noncommercial replication and distribution of the article with the strict proviso that no changes or edits are made and the original work is properly cited (including links to both the formal publication through the relevant DOI and the license). See: https://creativecommons.org/licenses/by-nc-nd/4.0/.

\section{References}

1. Rockson SG. Lymphedema after Breast Cancer Treatment. N Engl J Med 2018;379:1937-44.

2. Lucci A, McCall LM, Beitsch PD, et al. Surgical complications associated with sentinel lymph node dissection (SLND) plus axillary lymph node dissection compared with SLND alone in the American College of Surgeons Oncology Group Trial Z0011. J Clin Oncol 2007;25:3657-63.

3. He L, Qu H, Wu Q, et al. Lymphedema in survivors of breast cancer. Oncol Lett 2020;19:2085-96.

4. Cox K, Weeks J, Mills P, et al. Contrast-Enhanced Ultrasound Biopsy of Sentinel Lymph Nodes in Patients with Breast Cancer: Implications for Axillary Metastases and Conservation. Ann Surg Oncol 2016;23:58-64.

5. Kilbride KE, Lee MC, Nees AV, et al. Axillary staging prior to neoadjuvant chemotherapy for breast cancer: predictors of recurrence. Ann Surg Oncol 2008;15:3252-8.

6. O'Flynn EA, Wilson AR, Michell MJ. Image-guided breast biopsy: state-of-the-art. Clin Radiol 2010;65:259-70.

7. Rautiainen S, Masarwah A, Sudah M, et al. Axillary lymph node biopsy in newly diagnosed invasive breast cancer: comparative accuracy of fine-needle aspiration biopsy versus core-needle biopsy. Radiology 2013;269:54-60.

8. Kwon S, Price RE. Characterization of internodal collecting lymphatic vessel function after surgical removal of an axillary lymph node in mice. Biomed Opt Express 2016;7:1100-15.

9. Hall MA, Robinson H, Chan W, et al. Detection of lymphangiogenesis by near-infrared fluorescence imaging and responses to VEGF-C during healing in a mouse fulldermis thickness wound model. Wound Repair Regen 
2013;21:604-15.

10. Sweet DT, Jimenez JM, Chang J, et al. Lymph flow regulates collecting lymphatic vessel maturation in vivo. $\mathrm{J}$ Clin Invest 2015;125:2995-3007.

11. Mebius RE, Streeter PR, Breve J, et al. The influence of afferent lymphatic vessel interruption on vascular addressin expression. J Cell Biol 1991;115:85-95.

12. Kim T, Giuliano AE, Lyman GH. Lymphatic mapping and sentinel lymph node biopsy in early-stage breast carcinoma: a metaanalysis. Cancer 2006;106:4-16.

13. Proulx ST, Luciani P, Christiansen A, et al. Use of a PEGconjugated bright near-infrared dye for functional imaging of rerouting of tumor lymphatic drainage after sentinel lymph node metastasis. Biomaterials 2013;34:5128-37.

14. Suga K, Yuan Y, Okada M, et al. Breast sentinel lymph node mapping at CT lymphography with iopamidol: preliminary experience. Radiology 2004;230:543-52.

15. Zhou W, Zhao Y, Pan H, et al. Great tumour burden in the axilla may influence lymphatic drainage in breast cancer patients. Breast Cancer Res Treat 2016;157:503-10.

16. Houssami N, Ciatto S, Turner RM, et al. Preoperative ultrasound-guided needle biopsy of axillary nodes in

Cite this article as: Xia Y, Zheng M, Chen L, Cui Y, Huang H, Kong P, Zhou W, Xie H, Wang S. Changes of lymphatic flow caused by core needle biopsy of axillary sentinel lymph node in a rabbit model. Ann Palliat Med 2021;10(2):1480-1487. doi: 10.21037/apm-20-882 invasive breast cancer: meta-analysis of its accuracy and utility in staging the axilla. Ann Surg 2011;254:243-51.

17. Krishnamurthy S, Sneige N, Bedi DG, et al. Role of ultrasound-guided fine-needle aspiration of indeterminate and suspicious axillary lymph nodes in the initial staging of breast carcinoma. Cancer 2002;95:982-8.

18. Oude Ophuis CMC, Koppert LB, de Monye C, et al. Gamma probe and ultrasound guided fine needle aspiration cytology of the sentinel node (GULF) trial overview of the literature, pilot and study protocol. BMC Cancer 2017;17:258.

19. Giuliano AE, Ballman KV, McCall L, et al. Effect of Axillary Dissection vs No Axillary Dissection on 10-Year Overall Survival Among Women With Invasive Breast Cancer and Sentinel Node Metastasis: The ACOSOG Z0011 (Alliance) Randomized Clinical Trial. JAMA 2017;318:918-26.

20. Liang Y, Chen X, Zhan W, et al. Can Clinically NodeNegative Breast Cancer Patients with Suspicious Axillary Lymph Nodes at Ultrasound But Negative Fine-Needle Aspiration Be Approached as Having Node-Negative Disease? Ann Surg Oncol 2017;24:1874-80. 